Cite this: Metallomics, 2014 6, 1324

Received 7th March 2014, Accepted 14th May 2014

DOI: $10.1039 / c 4 m t 00069 b$

www.rsc.org/metallomics

\title{
Transition metal complexes with bioactive ligands: mechanisms for selective ligand release and applications for drug delivery
}

\begin{abstract}
Anna K. Renfrew
The unique properties of transition metal complexes, such as environment-responsive ligand exchange kinetics, diverse photochemical and photophysical properties, and the ability to form specific interactions with biomolecules, make them interesting platforms for selective drug delivery. This minireview will focus on recent examples of rationally designed complexes with bioactive ligands, exploring the different roles of the metal, and mechanisms of ligand release. Developments in the techniques used to study the mechanisms of action of metal-drug complexes will also be discussed, including X-ray protein crystallography, fluorescence lifetime imaging, and X-ray absorption spectroscopy.

.
\end{abstract}

\section{Introduction}

In recent years, drug development has evolved from serendipitous discoveries to design at the molecular level for the selective inhibition of a single enzyme. Clinical application of such rationally designed compounds has, however, met with limited success. While many new compounds are highly successful during in vitro and preclinical studies, half of all drug candidates that enter clinical trials fail due to problems such as poor bioavailability, low efficacy and severe side effects. ${ }^{1}$ Synthetic modifications

The University of Sydney, Chemistry, School of Chemistry, Building F11, Sydney, New South Wales, Australia. E-mail: anna.renfrew@sydney.edu.au

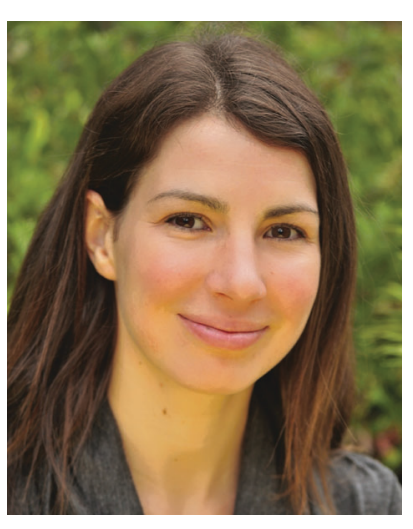

Anna K. Renfrew
Anna Renfrew was born in Edinburgh, Scotland and received her Masters in Chemistry from the University of York in 2006. In 2010, she received her $\mathrm{PhD}$ from the Ecole Polytechnique Federale de Lausanne under the supervision of Professor Paul Dyson, followed by a Swiss National Science Foundation postdoctoral fellowship at the University of Sydney under the mentorship of Professor Trevor Hambley. Since 2013, she has been carrying out at Discovery Early Career Researcher Award the University of Sydney. Her research interests are ruthenium-based anticancer agents and light-activated prodrugs. to overcome these limitations are often difficult and require a large number of synthetic steps. An alternative approach is to deliver the drug with a chaperone that encapsulates or complexes the drug. This is a synthetically simple strategy that can be an effective means of improving both the pharmacokinetic and pharmacodynamic properties of the parent drug. With a wide range of potential ligands and diverse synthetic chemistry, metal complexes represent versatile scaffolds that can be tailored to overcome the specific limitations of a broad spectrum of drugs.

\section{- Enhancing solubility and bioavailability}

Approximately $40 \%$ of drug candidates produced from combinatorial screening programmes have poor aqueous solubility (less than $10 \mu \mathrm{M}) .^{2}$ Coordination of a drug to a positivelycharged metal complex has been shown to greatly improve aqueous solubility in many cases: for example the proton pump inhibitor esomeprazole has very low aqueous solubility and is typically administered as esomeprazole magnesium, a water soluble bis(esomeprazole) magnesium complex. ${ }^{3}$ Alternatively, coordination of a metal to negatively-charged groups such as carboxylates and phosphates can reduce the negative charge of a drug, enhancing passive cellular uptake and absorption. ${ }^{4}$ In addition, endogenous metal ions and complexes can offer active transport pathways into the cell, allowing greater and more selective cellular accumulation of the drug.

\section{- Reducing systemic toxicity}

Transition metal complexes are particularly suited to controlled drug release, having bonds that are highly responsive to their environment. Prodrug complexes can be designed that are inert under normal physiological conditions, but become labile with a change in environment such as redox status, $\mathrm{pH}$ or the 
localised application of light. By deactivating a drug before it reaches its target, side reactions and premature metabolism that can lead to undesirable side effects can be reduced.

\section{- Increasing potency with multiple modes of action}

In addition to overcoming the limitations of an organic drug, the metal complex can itself be biologically active. By generating an active metal complex and active organic molecule from a single prodrug, multiple means of acting upon a target can be achieved. $^{5}$ Dual-action drugs may be more potent than the parent organic drug and be able to circumvent drug resistance mechanisms.

This review will present different applications of metal-drug chaperones across medicinal chemistry as delivery vehicles for anticancer, antidiabetic and antiparasitic agents, and neurochemicals. This review does not intend to be exhaustive; rather it aims to highlight some of the recent developments in rationally designed metal-drug complexes. Complexes where the role of the metal is purely structural and the mechanism of action does not involve release of a cytotoxic ligand have been well reviewed elsewhere and will not be covered here. ${ }^{6-9}$ We will give examples of drug candidates that have been unsuccessful in clinical trials or sub-optimal in clinical use and discuss how their limitations may be overcome through delivery in a metal chaperone complex. Different approaches towards selective drug release will be discussed, focusing on four mechanisms: (1) activation by reduction, (2) enzymatic activation, (3) light-triggered drug release, and (4) stimuli-responsive molecular cages.

\section{(1) Activation by reduction}

One of the distinctive features of many transition metal complexes is the large difference in lability between different oxidation states. This characteristic can be exploited in the design of prodrugs for selective activation in environments with a different redox state. Perhaps the most studied example is the hypoxic microenvironment found in large solid tumours, the result of a poor oxygen supply and elevated levels of reducing agents and reductase enzymes. A number of organic and metal-based prodrugs have been developed to target this environment, ${ }^{10}$ including several examples of metal chaperones complexes that are activated by reduction.

\section{Cobalt(III) hypoxia-activated chaperones}

Cobalt complexes are well suited for use as bioreductive prodrugs, having a very inert oxidised cobalt(III) state and very labile reduced cobalt(II) state. ${ }^{11}$ Bioreductive cobalt chaperones of a number of antitumour agents such as nitrogen mustards, ${ }^{12}$ curcumin ${ }^{13}$ and the matrix metalloproteinase inhibitor Marimastat ${ }^{14}$ have been reported. In each case, coordination of the organic drug to cobalt deactivates the cytotoxin, either by blocking the active site or coordinating to a lone pair, yielding an inert prodrug. In the absence of oxygen, the cobalt(III) centre is reduced to labile cobalt(II) and the active drug released through ligand exchange (Fig. 1). Alternatively, chaperones can be reduced in hypoxic tissue by clinically relevant doses of radiation, offering spatial and temporal control over drug release. ${ }^{15}$

The selectivity of a number of cobalt bioreductive prodrugs for hypoxic tumour cells has been evaluated in both in vitro and in vivo models. Remarkable in vitro hypoxia-selective activity observed for a series of cobalt(III) prodrugs containing the DNA minor groove alkylator (1-(chloromethyl)-5-hydroxy-1 $H$-pyrrolo$[3,2-f]$ quinolin-3(2H)-yl)(5,6,7-trimethoxy- $1 H$-indol-2-yl)methanone, which were found to be 81-212 times more cytotoxic under hypoxic conditions than normal oxygenated cell culture conditions. ${ }^{16}$ Despite this selective activity in vitro, the most potent of the series, complex 1, [1-(chloromethyl)-3-(5,6,7-trimethoxyindol-2ylcarbonyl)-2,3-dihydro- $1 H$-pyrrolo[3,2-f]quinolin-5-ato(1,4,8,11tetraazabicyclo[6.6.2] hexadecane)cobalt(III)], was not significantly effective against hypoxic cells in a HT29 xenograft model. Complex 2, ( $N$-[2,2-dimethyl-1-(methylcarbamoyl)propyl]-2-[hydroxy(hydroxycarbamoyl)methyl]-4-methyl-pentanamide)(tris(2-methylpyridyl)amine)cobalt(III), containing the broad spectrum matrix metalloproteinase inhibitor Marimastat, was well tolerated in a 4T1.2 mouse model and found to be more effective at reducing tumour size than free Marimastat: however, both Marimastat and 2 increased tumour metastases relative to the control. Both of these in vivo studies suggest that, while cobalt bioreductive
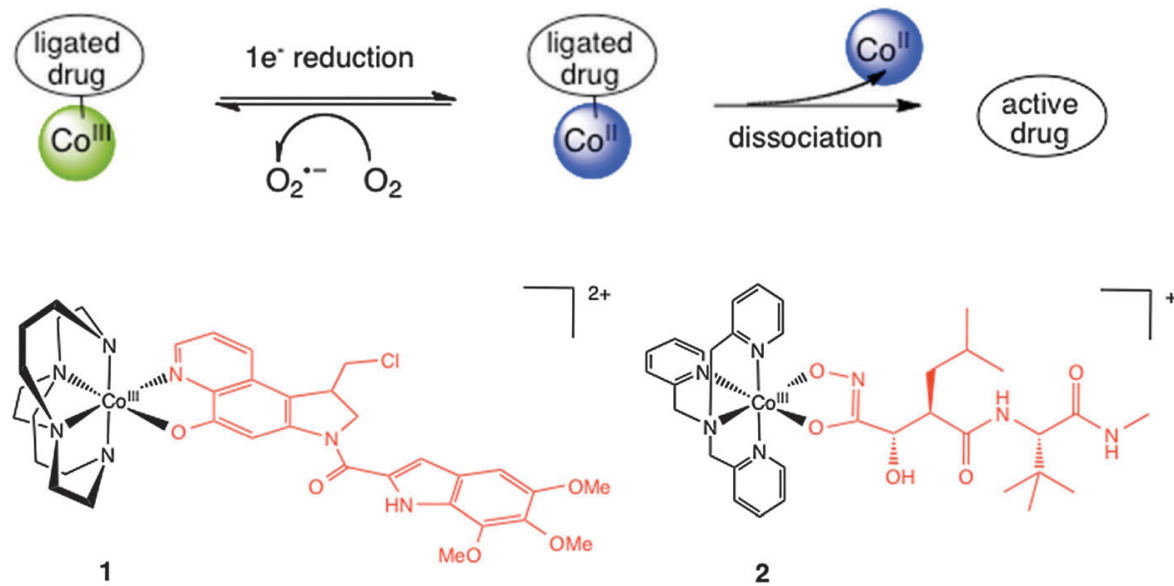

Fig. 1 (top) Proposed mechanism for the hypoxia-selectivity of cobalt(III) bioreductive prodrugs. (bottom) Examples of cobalt(III) complexes 1 and 2. 
prodrugs show promising behaviour in preliminary studies, a better understanding of their metabolism and fate is needed for the design of future complexes.

Ahn and coworkers have investigated possible pathways to reduction in vivo. They found no correlation between the expression of cytotochrome $\mathrm{P} 450$ reductase (the major one-electron reductase in mammalian cells) and the cytotoxicity or extent of reduction of cobalt(III) prodrugs, suggesting that other biological reductants such as ascorbate, thiols or $\operatorname{NADP}\left(\mathrm{H}^{+}\right)$may be involved. ${ }^{17}$ A recent study demonstrated the reaction of model cobalt(III) hydroxamic acid and $N$-hydroxy-pyridonone complexes with cysteine methyl ester to yield $\left[\mathrm{Co}(\mathrm{III})(\mathrm{CysOMe}-\mathrm{H})_{3}\right]$ as the final product. The authors saw no evidence for reduction of the cobalt centre, proposing that ligand exchange may also be a pathway to drug release in some cases. ${ }^{18}$ Spectroscopic and fluorescent probes have also been used to study the localisation and time scale of drug release in biological systems. The fluorescence of most fluorescent ligands is quenched when coordinated to cobalt(III), allowing release of the fluorophore to be observed in live cells by the return of fluorescence. Kim et al. used a three-dimensional tumour model with fluorescentlytagged hypoxic cells, to determine whether fluorescent coumarin and anthraquinone ligands are released from cobalt(III) complexes in the hypoxic cells. ${ }^{19}$ We have recently demonstrated the hypoxia-selective delivery and release of the anticancer drug curcumin by a cobalt(III) chaperone complex 3, [(1E,6E)-1,7bis(4-hydroxy-3-methoxyphenyl)-1,6-heptadiene-3,5-dione (tris(2methylpyridyl)amine)cobalt(III)]. Curcumin has been unsuccessful in a number of clinical trials due to its poor solubility and very short serum half-life, while the cobalt(III) prodrug is water soluble and stable for 24 hours in buffer. Using a combination of techniques, including X-ray absorbance near edge structure spectroscopy (XANES) and fluorescence lifetime imaging (FLIM), we were able to show that curcumin is released in hypoxic tumour cells where the cobalt(III) prodrug is reduced to cobalt(II) (Fig. 2). ${ }^{13}$

\section{Platinum(Iv) hypoxia-activated prodrugs}

In the case of cobalt bioreductive chaperones, the metal acts only as a carrier and is not required to exert any cytotoxic effects. Alternatively, platinum(Iv) hypoxia-activated prodrugs combine a cytotoxic metal complex with an organic anticancer agent so that reduction of the prodrug yields two complimentary active drugs. Platinum(II) anticancer drugs are currently used in the treatment of approximately $50 \%$ of solid tumours, though they are associated with a high incidence of drug resistance and side effects. ${ }^{20}$ Platinum(Iv) prodrugs were developed as a means of overcoming these limitations. Pt(Iv) complexes are six-coordinate, octahedral and inert, but undergo a two-electron reduction to a square planar Pt(II) complex, with loss of the two axial ligands. The advantages of the prodrug are two-fold: firstly the Pt(Iv) prodrug is more stable and the risk of premature side reactions is lessened; secondly the activation-by-reduction mechanism provides a degree of selectivity for hypoxic tumour regions. There are a number of examples of Pt(Iv) complexes with tumourtargeting groups as axial ligands, including oestrogen, ${ }^{21}$ bile acids, $^{22}$ and short peptides. ${ }^{23}$

Where both the axial ligands are anticancer agents, reduction of the $\mathrm{Pt}(\mathrm{Iv})$ prodrug yields an active $\mathrm{Pt}(\mathrm{II})$ complex and two equivalents of active ligand (Fig. 3). Lippard et al. have used this strategy with dichloroacetic acid (DCA) ligands in the axial position (4). ${ }^{24}$ DCA is an anticancer agent that can reverse the Warburg effect by inhibiting the enzyme pyruvate dehydrogenase kinase. By shifting cellular metabolism from glycolysis to glucose oxidation, DCA makes malignant cells more vulnerable to cell death programs without affecting normal cells. The authors were able to confirm the release of DCA in tumour cells by the change in membrane potential due to pyruvate dehydrogenase kinase inhibition. They also demonstrated the formation of intrastrand DNA cross links that are typical of cisplatin, revealing that the platinum-DCA complex has two killing modes: DCA disrupts mitochondrial function and cisplatin impedes DNA-mediated processes in the nucleus. Complex 4 was found to be more effective at inducing apoptosis than DCA or cisplatin alone, and also nontoxic in nontumourigenic cells where cisplatin was toxic. This is a promising system where both drugs protect the other from side reactions and also enhance each other's biological effect, though it should be noted that related platinum(Iv) cisplatin analogues with two carboxylate ligands are reduced by ascorbate under physiological conditions and 4 might not be sufficiently stable towards reduction for clinical applications. ${ }^{25,26}$

In a related strategy, Ang et al. developed a Pt(Iv) prodrug of cisplatin with two equivalents of the glutathione-transferase
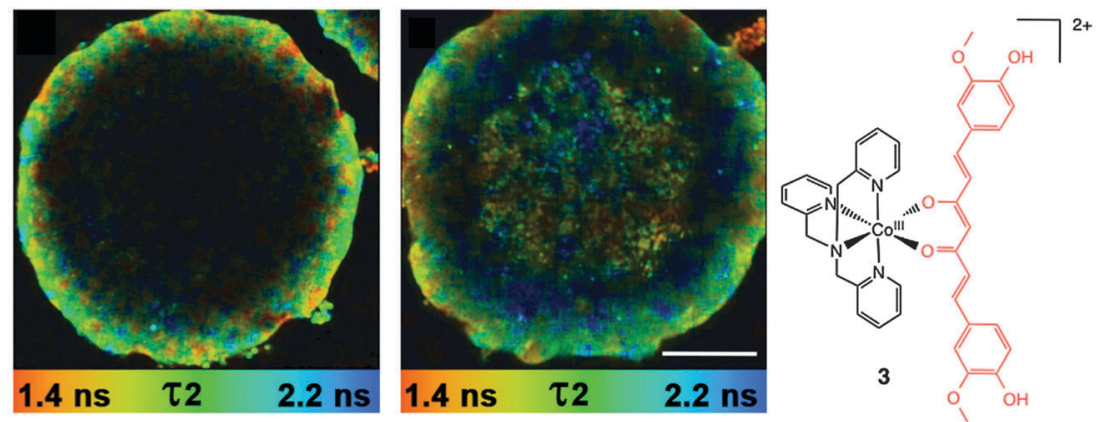

Fig. 2 False colour fluorescence lifetime images of curcumin (left) and $\mathbf{3}$ (right) in DLD-1 multicellular spheroids. Free curcumin only accumulates in the outer, oxygenated region of the spheroid while 3 penetrates to the inner hypoxic regions (scale bar $=100 \mu \mathrm{m}$ ). ${ }^{13}$ 

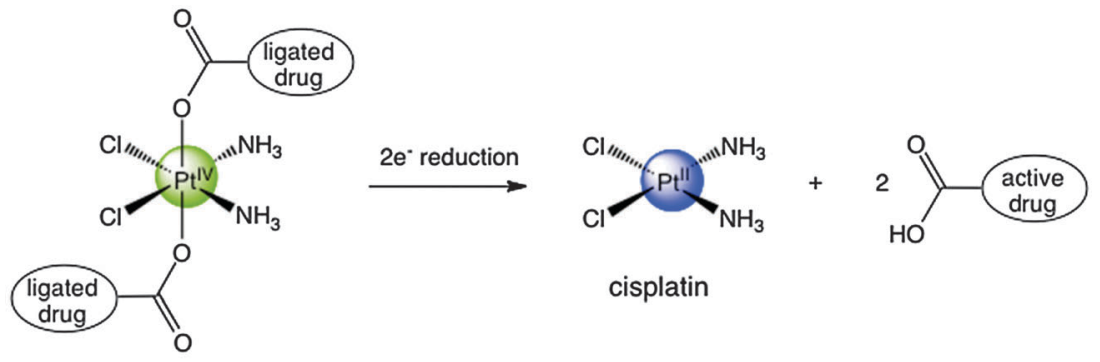

cisplatin

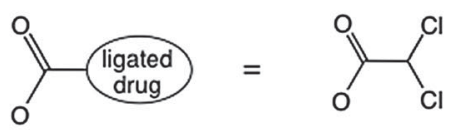

4, Dichloroacetic acid

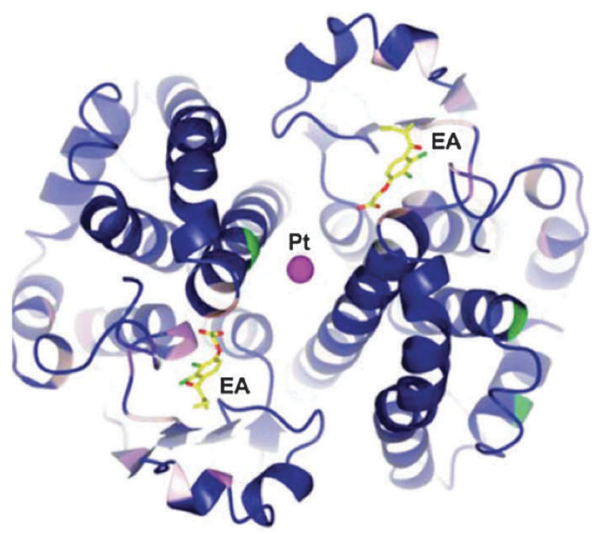

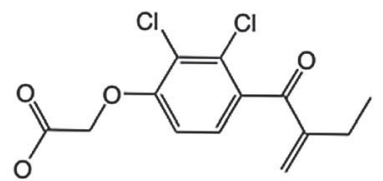

5, Ethacrynic acid

Fig. 3 (top) Proposed mechanism of activation for platinum(Iv) complexes $\mathbf{4}$ and $\mathbf{5}$. (bottom) Co-crystal structure of $\mathbf{5}$ bound to GST P1-1 with the ethacrynic acid ligands (EA) at the enzyme active site and platinum at the dimer interface. ${ }^{6}$

(GST) inhibitor, ethacrynic acid as the axial ligands. ${ }^{27}$ GST is involved in drug detoxification and is responsible for the cellular resistance of many drugs including cisplatin. Ethacrynic acid entered clinical trials in combination with a number of anticancer drugs but was unsuccessful due to the large difference in cellular uptake rates between ethacrynic acid and the drugs. By combining ethacrynic acid and a platinum drug in a single molecule, this issue may be circumvented. Complex 5 was more cytotoxic towards a small panel of cell lines than cisplatin or ethacrynic acid alone. The complex was also ten times more effective at inhibiting GST than free ethacrynic acid. Unexpectedly, a crystal structure of GST with $\mathbf{5}$ showed that the intact complex binds to the protein via the two ethacrynic acid molecules (Fig. 3). This suggests that the platinum centre of $\mathbf{5}$ may be scavenged by GST, though in a cellular environment it is likely that a significant proportion of $\mathbf{5}$ would be reduced before reaction with GST as $\mathrm{Pt}(\mathrm{Iv})$ complexes with a similar coordination sphere to $\mathbf{5}$ have been shown to undergo rapid intracellular reduction. ${ }^{28}$

It should be noted that a number of platinum(II) analogues of cisplatin and transplatin with bioactive ligands have also been prepared, in many cases exhibiting superior in vitro cytotoxicity to both the free ligand and original platinum complex. ${ }^{29,30}$

\section{(2) Enzymatic activation}

While DNA was considered the primary target of metal-based drugs for many years, there is a growing interest in complexes that can target enzymes and proteins. ${ }^{31}$ A number of metal complexes with bioactive ligands have been prepared where either the ligand or the metal can interact with a specific enzyme, leading to dissociation of the prodrug complex. Here we will focus on examples where the activating enzyme is overexpressed in, or unique to, the target cells.

\section{Organometallic ruthenium(II) complexes}

Organometallic ruthenium(II) arene complexes are an emerging class of metal-based drug with encouraging anticancer, antimetastatic, antibiotic, and antiparasitic activity. ${ }^{32-35}$ While the mechanisms of action of this class of compound are not well understood, interactions with specific enzymes are thought to play an important role in many cases. For the ruthenium complex to bind coordinatively to a biological target, it must undergo exchange of a mono or bidentate ligand. Where one of these ligands is biologically active, an active ruthenium complex and organic drug are released concurrently. A range of biologically active ligands have been investigated for this purpose, including monodentate imidazole-based ligands and bidentate $O, O$ - and $N, N$-chelating ligands (Fig. 4). 


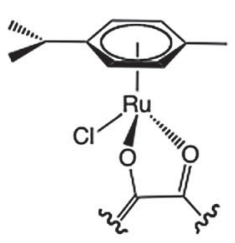<smiles>CCC(C)C(=O)O</smiles>

6, flavonoid topoisomerase(II) inhibitor<smiles>CC(C)=CCC1=C(O)C(=O)c2ccccc2C1=O</smiles>

\section{7, Lapachol}<smiles>[Z20]C(=O)O[R10](=O)OCO</smiles>

9, Ketaconazole

8, Ethacrynic acid

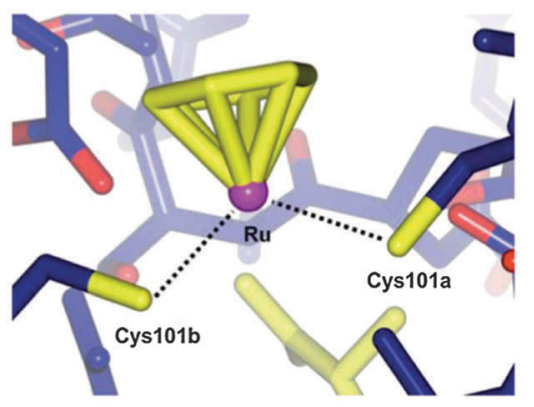

Fig. 4 (top) Examples of ruthenium(॥)arene complexes with bioactive ligands. (bottom) A ruthenium-ethacrynic acid complex bound to GST P1-1 through the two cysteine 101 residues of the individual monomers. ${ }^{6}$

Hartinger and coworkers have investigated ruthenium(II) arene complexes with bidentate flavonoid ligands (6). ${ }^{36}$ Flavonoids present an interesting opportunity for drug delivery as they have diverse anticancer and antioxidant properties but are often limited by poor aqueous solubility and bioavailability. The authors prepared a range of ruthenium complexes containing flavonoid inhibitors of topoisomerase II, an enzyme overexpressed in many types of cancer. They aimed to develop a compound where the flavonoids would first bind to the topoisomerase enzyme, leaving the ruthenium complex with two free coordination sites to form a covalent bond to DNA. The ruthenium-flavonoid complexes were around 10-fold more water-soluble than the free ligands and remained intact in solution for several days. Both the topoisomerase(II) inhibition and cytotoxicity of the ruthenium-flavonoid complexes were superior to those of the free flavonoids. The ruthenium complexes were also found to covalently bind catenated kinetoplast DNA in the presence of topoisomerase(II) though further studies are needed to confirm the simultaneous interaction of the complex with DNA and the enzyme.

In a second approach, the authors developed organometallic ruthenium(II), osmium(II) and rhodium(III) complexes of the naturally occurring flavonoid lapachol. ${ }^{37}$ Lapachol induces apoptosis through the production of reaction oxygen species (ROS) and has undergone several clinical trials as an anticancer agent, though the trials were discontinued due to toxicity.
Given that organometallic complexes are generally well tolerated in vivo, coordination of the drug to a metal complex may offer a means of reducing its systemic toxicity. In particular, the ruthenium(II) arene complex 7 was more effective at inducing apoptosis through production of ROS than the free drug, suggesting a synergistic effect between the ruthenium complex and flavonoid ligand. While it is not clear if or how the flavonoid ligand is released, a model protein binding study in which the complex was incubated with ubiquitin showed the formation of a ruthenium-ubiquitin adduct and release of free lapachol, suggesting a possible mechanism for intracellular release of the drug.

Analogues of the antimetastatic ruthenium arene complex RAPTA-C have been prepared with a range of biologically active ligands including a phenoxazine-based multi-drug resistance inhibitor, ${ }^{38}$ naphthalimide DNA intercalator, ${ }^{39}$ the glycolysis inhibitor lonidamine. ${ }^{40}$ and glutathione-transferase (GST) inhibitor, ethacrynic acid, tethered to an arene or imidazole ligand (8). ${ }^{41}$ A number of ruthenium(II) and osmium(II) arene complexes with cyclin-dependent kinase (CDK) inhibitors, ${ }^{42,43}$ aromatase inhibitors, ${ }^{44}$ and antibacterial quinolones ${ }^{45}$ as ligands have also been reported, many with interesting anticancer properties, though in these cases it is not clear whether cytotoxicity is due to the intact complex or release of the bioactive ligand.

In addition to anticancer activity, organometallic rutheniumimidazole complexes were found to be effective in the treatment of the parasitic diseases leishmania major and trypanosoma cruzi. ${ }^{46,47}$ 
The imidazole-containing drugs ketoconazole and clotrimazole reduce parasitic activity by inhibiting the cytochrome $\mathrm{P} 450$ dependent C-14 $\alpha$-demethylation of lanosterol to ergosterol. Ruthenium(II) arene complexes of ketaconazole (9) and clotrimazole exhibited higher antiparasitic activity than the free drug, but were less toxic towards human cells. The authors propose that the ruthenium complexes mask the toxicity of the imidazole as they are coordinated through the $\mathrm{N}(3)$ nitrogen of the imidazole group, which is required for binding to cyP $450_{\mathrm{DM}}$. The ruthenium product formed after loss of the imidazole ligand was also found to covalently bind to DNA, which may explain the increased antiparasitic activity of the ruthenium-imidazole complexes with respect to the parent drugs.

\section{A dual-action gold(I) complex}

Gold(I) phosphine complexes are most commonly used in the treatment of arthritis and inflammation, but are being increasingly studied for their anticancer activity, with covalent binding to disulphide reductases such as cathepsins and thioredoxin reductase (TrxR) implicated in their mode of action. X-ray crystallography and mass spectrometry studies on the interaction of gold phosphine complexes with reductase enzymes indicate that the active species is the gold ion itself, with the ligands only influencing the biodistribution and kinetics of the complex. ${ }^{48}$ In a recent example, a naphthalimide DNA intercalator was tethered to a gold(I) phosphine complex (10) with the rationale that TrxR binding would result in the release of the active naphthalimide ligand giving the complex two modes of action. ${ }^{49}$ The naphthalimide-gold complex was found to be good inhibitor of TrxR, and ESI mass spectrometry studies with a cysteine peptide mimic of the TrxR active site shows that gold covalently binds the cysteine residue with loss of the naphthalimide ligand. In vitro studies indicated that the mode of action of the complex involves inhibition of TrxR and induction of apoptosis via the mitochondrial pathway. Confocal fluorescence microscopy and atomic absorption spectroscopy revealed high levels of both the naphthalimide moiety and gold in the nucleus. Unexpectedly, 10 also exhibited significant anti-angiogenic activity in developing zebrafish embryos, which was not observed for similar gold complexes or the naphthalimide ligand alone.

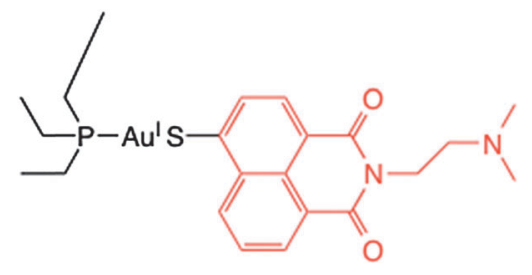

10

\section{Vitamin $B_{12}$-an endogenous metal complex}

The ruthenium and gold complexes discussed above are synthetic complexes designed to be compatible with physiological conditions. An alternative approach is to use a naturally occurring metal complex. An endogenous metal complex has the advantage of being well tolerated, and can potentially offer selective uptake mechanisms into the cell. One such complex is the cobalt-containing vitamin $\mathrm{B}_{12}$. As a water-soluble, non-toxic vitamin, with an axial coordination site available for drug conjugation, $\mathrm{B}_{12}$ is an attractive drug chaperone. To date, its application has been limited by the low uptake capacity ( $c a .2 .5 \mu \mathrm{g}$ per day for an adult), however, a number of recent advances, including haptocorrin-specific targeting and polymeric carriers, are going someway to overcoming these issues. ${ }^{50}$ When $\mathrm{B}_{12}$ is converted to its biologically active form, adenosylcobalamin, the cobalt centre is reduced via a three-step process from $\mathrm{Co}$ (III) to $\mathrm{Co}(\mathrm{I})$. During the reduction from $\mathrm{Co}(\mathrm{III})$ to $\mathrm{Co}(\mathrm{II})$, the cobalt releases its upper axial ligand. Where a drug is tethered to the axial ligand, this step offers a mechanism for intracellular drug release. A number of conjugates with different therapeutics at the upper axial position have been investigated (Fig. 5).

Alberto et al. exploited the increased demand of rapidly proliferating cancer cells for $\mathrm{B}_{12}$ for the delivery of cisplatin analogues. The authors prepared a series of $\mathrm{B}_{12}$-platinum(II) conjugates, where the cobalt(III) centre is bridged via a cyano ligand to a cisplatin analogue (11). ${ }^{51,52}$ The cyano-bridged conjugates were found to be stable in serum for 24 hours, while cisplatin has a serum half-life of 30 minutes. Using an in vitro adenosylation assay from salmonella enteric, the authors were able to show that the $\mathrm{B}_{12}-\mathrm{Pt}(\mathrm{II})$ conjugates are transported into the cell by receptor-mediated endocytosis via the transcobalamin II receptor. ${ }^{53}$ Subsequent release of the platinum complexes occurs inside the cell after enzymatic reduction of $\mathrm{Co}(\mathrm{III})$ to $\mathrm{Co}$ (II). These results demonstrate that the structure of the platinum-conjugated $\mathrm{B}_{12}$ can still be recognized by the same enzymes and undergo the same enzymatic reductions as the native vitamin. In addition, the platinum complexes undergo rapid reaction with deoxyguanosine in a manner similar to cisplatin following release from the $\mathrm{B}_{12}$ carrier. While the in vitro cytotoxicities of the $\mathrm{B}_{12}-\mathrm{Pt}$ (II) conjugates were lower than cisplatin alone, possibly due to the uptake limitation of $\mathrm{B}_{12}$, it should

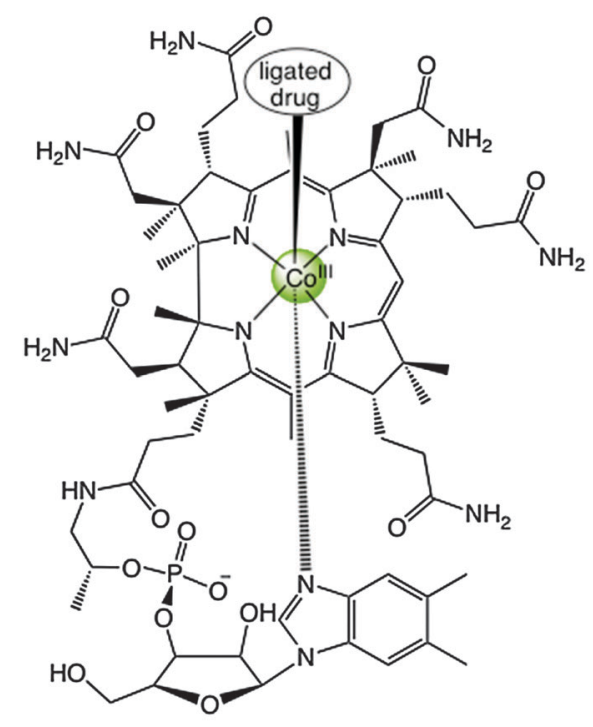<smiles>NP(N)(Cl)=NCc1ccccc1</smiles>

11

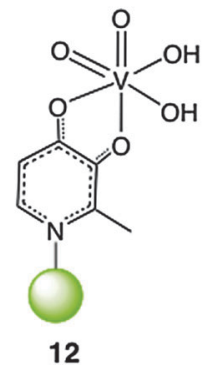

Fig. 5 Structure of vitamin $B_{12}$ and examples of a platinum anticancer drug (11) and vanadium antidiabetic complex (12) that have been conjugated to the upper axial ligand. 
be noted that the conjugate-complexes are much more stable in serum than cisplatin and could be more effective in vivo.

This strategy has recently been extended to the combined delivery of an organic and platinum-based anticancer agent by the same molecule. ${ }^{54}$ The clinically approved drugs cytarabine, dacarbazine, and anastrozole were attached to the platinum- $\mathrm{B}_{12}$ conjugates at the $\mathrm{Pt}$ (II) centre. The drug-conjugates were stable for days under physiological conditions and retained their affinity for $\mathrm{B}_{12}$ transport proteins. Reduction to $\mathrm{Co}$ (II) induced the release of the drug-bound $\mathrm{Pt}$ (II) complexes, followed by release of the active organic drug from the $\mathrm{Pt}(\mathrm{II})$ complex through ligand exchange. The $\mathrm{IC}_{50}$ values of the prodrugs were much higher than the free drug but when the prodrugs were chemically reduced first, the values were comparable.

In addition to the delivery of anticancer agents, vitamin $B_{12}$ has been investigated for the delivery of antidiabetic drugs. Two vanadium(v) complexes with insulin enhancing properties were conjugated to $\mathrm{B}_{12}$ via a 3-hydroxy-2-methyl-1-propyl-1 $H$-pyridin4-one linker (12). ${ }^{55}$ Both the absorption and cellular uptake of the conjugates were found to be superior to the free vanadium drugs. Accordingly, the $\mathrm{B}_{12}$-vanadium complexes were more effective at lowering blood glucose levels in a rat model than the vanadium complexes alone.

\section{(3) Photo-triggered drug release}

The chaperone complexes presented in the previous section rely on an intrinsic feature of the target cell or tissue for activation. Lightactivated chaperones are an attractive alternative, offering spatial and temporal control over the release of the active drug. Many inert transition metal complexes, particularly those with $\mathrm{d}^{3}$ and $\mathrm{d}^{6}$ electronic configurations, undergo ligand dissociation when excited by visible light of a suitable wavelength, and a diverse range of complexes that are cytotoxic in combination with light have been investigated. ${ }^{56}$

\section{Ruthenium(II) polypyridyl complexes}

Rather than using light to generate a reactive metal complex, a strategy developed by Etchenique et al. uses metal complexes as photolabile protecting groups to cage bioactive molecules (Fig. 6). The group used a ruthenium polypyridyl complex (13) to release two equivalents of 4-aminopyridine (4-AP), a neurochemical that acts as a potassium channel blocker. ${ }^{57}$ The biological activity of this prodrug was measured in a leech ganglion, and light irradiation was found to induce a neural response characteristic of 4-aminopyridine release. This idea has been expanded to include a range of photocaged neurotransmitters including glutamate, $\gamma$-amino butyric acid (GABA), serotonin, and tryptamine. ${ }^{58}$ Notably, the ruthenium-glutamate complex releases glutamate in less than $50 \mathrm{~ns}$ when excited at $532 \mathrm{~nm}$, offering both faster glutamate release and longer activation wavelengths than any reported organic photolabile protecting group. Both photocaged glutamate and 4-AP can be released by two-photon activation, meaning that much longer activation wavelengths ( $c a .800 \mathrm{~nm}$ ) can be employed. ${ }^{59,60}$ This is particularly important for clinical applications, where light of $>600 \mathrm{~nm}$ is desirable for good tissue penetration and minimal tissue damage. Ruthenium photocaged 4-AP, glutamate, GABA, and nicotine have recently become commercially available.

The same concept has been used to produce the first visible light photo-triggered gene inducer. The authors treated a culture of $E$. coli cells with complex 14, which contains the gene inducer methylthiogalactoside (MTG), bound to ruthenium through a thioether. ${ }^{58}$ Illumination of the cells with a LED-powered pocket projector induced the localised release of MTG, indicated by formation of a blue precipitate due to the hydrolysis of the substrate X-Gal. While MTG does not have any therapeutic applications, it could be substituted with other thioether containing genes, allowing the possibility to selectively induce genes in some cells and not others with applications in biotechnology and tissue engineering.

Turro and coworkers have explored the use of ruthenium polypyridyl complexes for the light-activated release of anticancer therapeutics. The authors have investigated the caging and light-induced release of two nitrile-based drugs, a peptidomimetic cathepsin $\mathrm{K}$ inhibitor and 5-cyanouracil, an inhibitor of pyrimidine catabolism. A series of complexes containing two

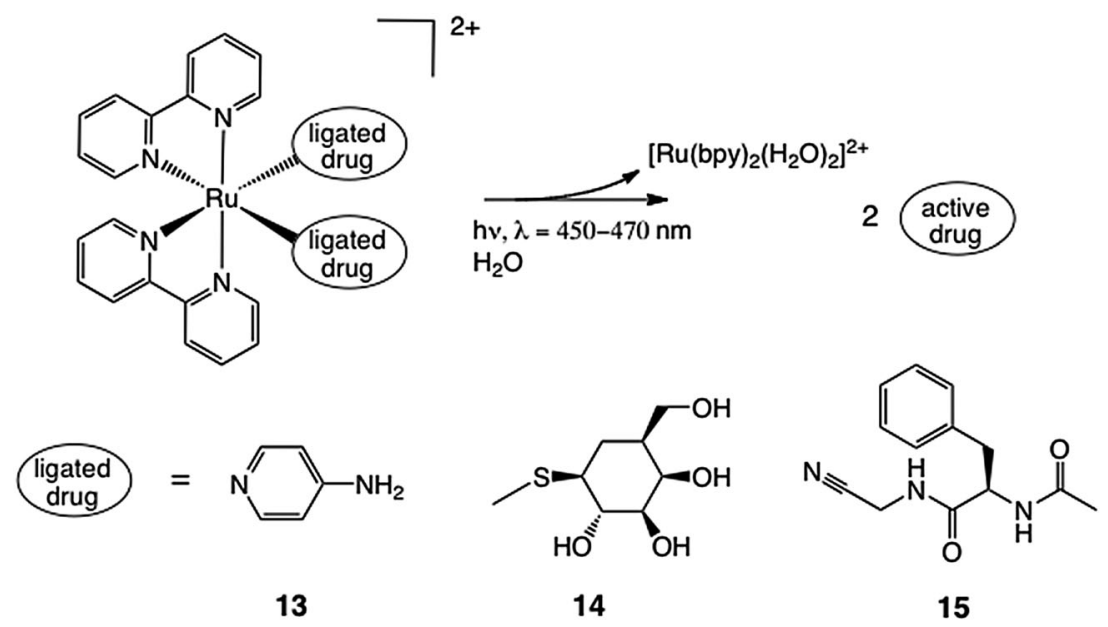

Fig. 6 Examples of ruthenium polypyridyl complexes investigated for photo-triggered drug release. 
or three equivalents of 5-cyanouracil were prepared and found to be stable in water in the dark. ${ }^{61,62}$ Irradiation with light $(\lambda>395 \mathrm{~nm})$ for 15 minutes lead to the release of two equivalents of 5-cyanouracil and one equivalent of an aquated ruthenium complex. Interestingly, only the axial nitrile ligands were found to undergo photoaquation. The bis(aqua) photoproducts were found to decrease the mobility of linearised plasmid DNA, indicative of covalent binding. Furthermore, one of the complexes decreased DNA mobility in combination with red light $(\lambda>645 \mathrm{~nm})$, within the optimum photodynamic therapy window of 600-850 $\mathrm{nm}$. The concurrent release of a covalent DNA binder and a fluorouracil analogue is promising for the development of dual action photochemotherapeutics. However, in vitro cytotoxicity studies suggest that only one equivalent of 5-cyanouracil is released on irradiation and give no evidence that the ruthenium photoproduct is binding to DNA or contributing to cell death. This may be the result of incomplete photolysis as only the bis(aqua) complexes are able to bind DNA, or the ruthenium complexes may not be reaching the cell nucleus.

In a subsequent example, the authors demonstrated the light-induced release of two equivalents of a nitrile-containing peptidomimetic cathepsin $\mathrm{K}$ inhibitor from a $\left[\mathrm{Ru}(\mathrm{bpy})_{2} \mathrm{~L}_{2}\right]^{2+}$ prodrug (15). ${ }^{63}$ The ability of the complex to inhibit cathepsin $\mathrm{K}$ increased 33 -fold where the solution was irradiated with visible light for 15 minutes. Inhibition of cathepsin B, an enzyme linked to cancer progression, also increased 14-fold with light treatment. Furthermore, in DU145 prostate cancer and hBMSC bone marrow stromal cell lysates, inhibition of cathepsin B was up to 8 times higher in the light-treated sample.

\section{Photocytotoxic curcumin complexes}

Chakravarty and coworkers have investigated light-activated prodrug complexes of curcumin. The photocytotoxicity of free curcumin has been reported in a number of in vitro and in vivo models; however, further development in this area is limited by the issues presented above, e.g. the poor stability and bioavailability of curcumin. The authors demonstrated that coordination of curcumin to an oxovanadium(Iv) dipyridophenazine (dppz) complex (16) prevents the hydrolytic cleavage of curcumin under physiological conditions. ${ }^{64}$ The vanadiumcurcumin complex is non-toxic towards HeLa cells in the dark up to a concentration of $50 \mu \mathrm{M}$ (the solubility limit), but has an $\mathrm{IC}_{50}$ value of $3.3 \mu \mathrm{M}$ when treated cells are irradiated with visible light. The $\mathrm{IC}_{50}$ of free curcumin decrease 10-fold in combination with light, from 85 to $8 \mu \mathrm{M}$. As vanadiumdppz complexes are photocytotoxic through light-activated DNA cleavage, the complexes can increase the potency of curcumin both by protecting it from hydrolysis prior to photoactivation and by introducing a complimentary photocytotoxic effect through DNA cleavage. Confocal fluorescence microscopy also shows much higher levels of fluorescence from cells treated with the complex than those treated with the free drug, suggesting that the vanadium carrier enhances uptake of the drug, or prevents its metabolism to a nonfluorescent product. Lanthanoid and copper(II) curcumin complexes have also been shown to exhibit photo-selective DNA cleavage and cytotoxicity. ${ }^{65,66}$

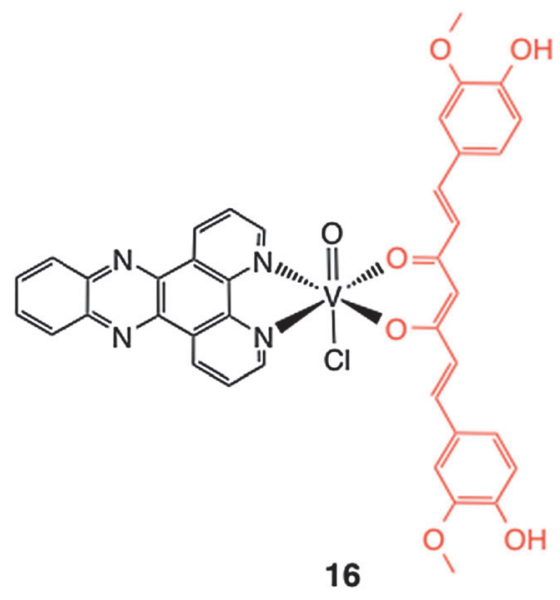

\section{Light-triggered release of gases}

In addition to organic drugs and neurochemicals, the diatomic gases nitric oxide and carbon monoxide are interesting candidates for delivery. Both gases are produced endogenously and are essential to a number of signalling processes but are toxic at higher concentrations. Due to the complex biological properties of gas molecules, a high degree of control over the dose of drug released is essential.

Nitric oxide (NO) is involved in a number of physiological and pathological processes including blood pressure control, neurotransmission, inhibition of platelet aggregation, and immunological responses. ${ }^{67}$ At higher concentrations, NO is cytotoxic and therefore selective release of NO can be used for the treatment of cancer and other diseases. Targeted doses of NO can also be applied to initiate an immune response to combat infection. In spite of the biological importance of NO, its application is limited by high reactivity and a short half-life, necessitating the use of a prodrug for the delivery of NO. A number of metal nitrosyl complexes have been investigated for this purpose. ${ }^{68}$ The majority use light-activation as a trigger for NO release though activation by enzymes and reduction have also been studied. Mascharak and coworkers have made significant advances in this field, developing a number of ruthenium and manganese nitrosyl complexes with pyridine carboxamide ligands, including the first metal nitrosyl that can be activated by wavelengths of up to $900 \mathrm{~nm} .{ }^{69}$ Another class of NO donor, iron-sulphur nitrosyl clusters developed by Ford et al., can be activated by two-photon activation or lanthanoid-based upconverting nanoparticles to release $\mathrm{NO}$ at near infrared wavelengths. ${ }^{70}$ Nitrosyl complexes have also been incorporated into hydrogel bandages and powders. This allows the prodrug to be applied directly to the surface of a wound prior to activation and is proposed as a method to selectively kill bacteria in wounds. ${ }^{71}$

Carbon monoxide (CO) has a number of biological roles, acting as a messenger, vasodilator, and cytoprotectant. There is interest in the therapeutic application of $\mathrm{CO}$ to reduce inflammation, suppress organ graft rejection and treat cancer, however, 


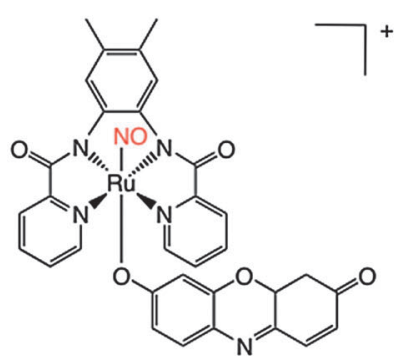

17<smiles>CC(C)C[C@H](NC(=O)[C@H](Cc1ccccc1)NC(=O)CNC(=O)CNC(=O)[C@H](Cc1ccc(O)cc1)NC(=O)CCC(=O)c1ccccc1)C(=O)O</smiles>

18

Fig. 7 Examples of metal prodrug complexes for NO (17) and CO (18) delivery.

as the molecule is toxic at high concentrations, controlling the target and dose of $\mathrm{CO}$ is particularly important. Most carbon monoxide releasing molecules (CORMS) release CO through aquation and typically have a short half-lives and release a high concentration of $\mathrm{CO}$ before reaching their target. By using light to cleave a metal-CO bond, highly inert CORMS can de developed that do not release $\mathrm{CO}$ prior to light treatment. ${ }^{72,73}$ Schatzschneider and coworkers have developed a series of manganese $(\mathrm{I})$ tricarbonyl complexes that release one or two equivalents of $\mathrm{CO}$ when irradiated at $365 \mathrm{~nm}^{74}$ While the activation wavelength is shorter than desirable for biological applications, the high stability of the complexes in the dark and ease of functionalisation with targeting peptides are very promising for the development of highly selective CORMS (Fig. 7).

\section{Molecular cages}

There is growing interest in the use of macromolecular metal complex structures such as metal-organic frameworks, liposomes, dendrimers, metalla-cages, and nanoparticles that can encapsulate and deliver a drug. These large systems are particularly interesting for the delivery of anticancer agents as they can potentially accumulate preferentially in tumour tissue due to the enhanced permeability and retention (EPR) effect. ${ }^{75}$ Nanoscale carriers such as metal-organic frameworks and nanoparticles are outside of the scope of this review and have been thoroughly reviewed elsewhere. ${ }^{76-79}$ Instead, this section will focus on small supramolecular structures with up to eight metal centres (Fig. 8).

Therrien et al. first explored the use of molecular cages for drug delivery with a series of organometallic ruthenium(II) metallaprisms. ${ }^{80}$ The metalla-prisms have a polar exterior and hydrophobic core and are able to encapsulate hydrophobic drugs to yield a water-soluble host-guest complex, allowing poorly soluble drugs to be prepared in aqueous media. The size of the hydrophobic cavity can be controlled to accommodate drugs of different sizes $^{81}$ and a range of different molecules have been encapsulated including cytotoxic platinum(II) and palladium(II) complexes, functionalised pyrenes, ${ }^{82}$ and photosensitisers. ${ }^{83}$ In each case, the drug-complexes were up to 10-fold more cytotoxic than the free prisms or drugs. Using confocal fluorescence microscopy and flow cytometry, the authors demonstrated that a fluorescent pyrene is released intracellularly from its host complex and that the intracellular pyrene concentration increases 10 -fold when the fluorophore is encapsulated in a prism. ${ }^{84}$ The cellular

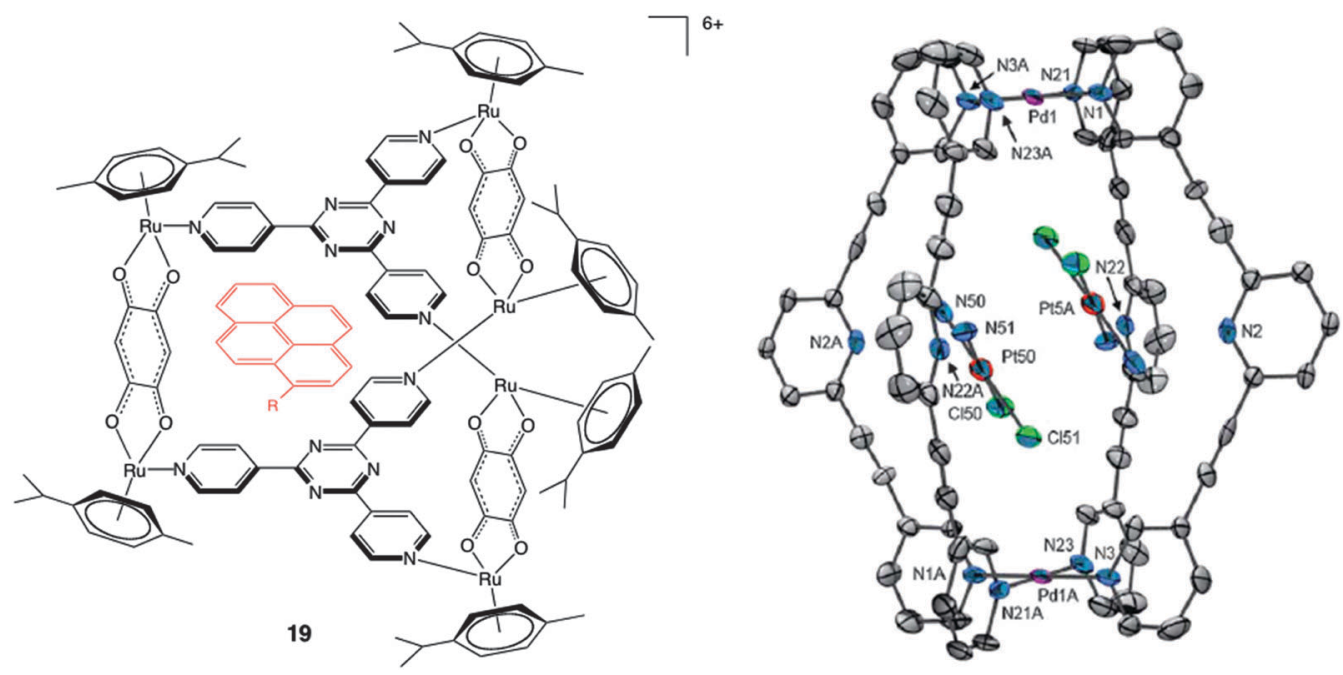

Fig. 8 (left) Example of a ruthenium metalla-prism with a hydrophobic pyrene in its interior. (right) Crystal structure of dipalladium cage $\mathbf{2 0}$ with two cisplatin molecules encapsulated. ${ }^{86}$ 
uptake of the metalla-prisms is thought to occur via endocytosis, and drug release through disassembly of the cages by reaction with certain amino acids and glutathione. ${ }^{85}$ The metallaprisms can improve the biological profile of a hydrophobic drug in several ways: rendering it soluble in aqueous media, actively transporting it into the cell and significantly increasing its cellular concentration.

In a related approach, Crowley and coworkers have developed dipalladium(II) cages capable of encapsulating two equivalents of cisplatin (20). The structure of host-guest adduct was confirmed by NMR solution studies, mass spectrometry and X-ray crystallography. In the presence of a competing ligand, the cage was disassembled, and both cisplatin molecules released. ${ }^{86}$ Though this prototype cage is not sufficiently stable in aqueous media to be used for biological applications, the design of a stimuliresponsive cage is an interesting approach towards selective drug release.

\section{Conclusions}

The metal chaperones highlighted in this review demonstrate the various ways in which a prodrug complex can improve and enhance the properties of a parent drug. The examples presented emphasise the versatility of this strategy, covering a broad range of structurally different drugs for the treatment of cancer, diabetes, neurochemicals disorders, parasitic diseases and bacterial infections. Considering the diverse synthetic chemistry of transition metal complexes, there is enormous potential to expand this concept, and with synthetic methods for the conjugation of targeting groups to metal complexes becoming increasingly robust, ${ }^{87,88}$ the prospect of highly selective chaperone complexes, tailored for the delivery of an individual drug, is becoming increasingly viable.

Most recent developments in drug delivery have focused on macromolecular chaperones such as liposomes, dendrimers and nanoparticles. However, a number of potential limitations with these macromolecular delivery systems have been reported, including difficulty in obtaining well-defined particle size, poor solubility, slow degradation leading to accumulation in the liver and other organs, and potentially fatal side effects such as cardiovascular damage, blood clots and hypersensitivity reactions. ${ }^{89,90}$ As a well-defined, small molecule drug delivery system, metal complexes are not subject to most of these limitations and have the advantage of not significantly altering the size of the parent drug. While further studies are required to determine the possibility of toxic side effects from the metal complex or metabolite, the in vivo studies conducted with hypoxia-activated cobalt(III) and light-activated ruthenium(II) chaperones showed that the complexes are well tolerated in the mouse or leech models. In certain cases, such as the platinum(Iv) or ruthenium(II)arene complexes, the metal complex is designed to exert its own cytotoxic effect once the prodrug complex has been activated.

Despite the promising preliminary activity observed for many of the complexes presented, there remain a number of challenges in developing chaperone complexes suitable for clinical applications. A major consideration is the stability of the prodrug under physiological conditions, where premature reduction and interactions with biomolecules can lead to the non-selective release of a drug. This is particularly relevant for the chaperone complexes that are activated by interactions with specific enzymes. In this respect, activation by light may prove to be the most promising approach, allowing the use of highly inert complexes that are only activated where a light source has been applied. While the application of such compounds is currently limited to regions that can be penetrated by a light, advances in light sources such as two-photon activation and next generation photodynamic therapy are increasing the range of tissues that can be targeted.

The few studies that have been conducted in animal models also demonstrate that the activity of these prodrug complexes in vivo may be markedly more complicated than for simple in vitro models. As such, the development of techniques to study metal-based drugs in biological systems is crucial to the evaluation of this class of compound compounds. This review has highlighted the use of several techniques to explore the distribution and fate of a metal complex or fluorescent drug in cells, including fluorescence lifetime imaging, flow cytometry and X-ray absorption spectroscopy. While not offering a complete picture, in combination these techniques can provide valuable insights into the localisation of a fluorescent drug, and the oxidation state and coordination environment of a metal complex. Such information may facilitate the design of future derivatives and direct the progression of lead compounds towards clinical applications.

\section{Notes and references}

1 J. Hodgson, Nat. Biotechnol., 2001, 19, 722-726.

2 J. Rautio, H. Kumpulainen, T. Heimbach, R. Oliyai, D. Oh, T. Jarvinen and J. Savolainen, Nat. Rev. Drug Discovery, 2008, 7, 255-270.

3 T. J. Johnson and D. D. Hedge, Am. J. Health-Syst. Pharm., 2002, 59, 1333-1339.

4 B. Demoro, F. Caruso, M. Rossi, D. Benitez, M. Gonzalez, H. Cerecetto, B. Parajon-Costa, J. Castiglioni, M. Galizzi, R. Docampo, L. Otero and D. Gambino, J. Inorg. Biochem., 2010, 104, 1252-1258.

5 J. E. Weder, C. T. Dillon, T. W. Hambley, B. J. Kennedy, P. A. Lay, J. R. Biffin, H. L. Regtop and N. M. Davies, Coord. Chem. Rev., 2002, 232, 95-126.

6 K. J. Kilpin and P. J. Dyson, Chem. Sci., 2013, 4, 1410-1419.

7 S. P. Mulcahy and E. Meggers, Top. Organomet. Chem., 2010, 32, 141-153.

8 A. Vessieres, S. Top, W. Beck, E. Hillard and G. Jaouen, Dalton Trans., 2006, 529-541.

9 U. Schatzschneider and N. Metzler-Nolte, Angew. Chem., Int. Ed., 2006, 45, 1504-1507.

10 W. A. Denny, Future Oncol., 2010, 6, 419-428.

11 M. D. Hall, T. W. Failes, N. Yamamoto and T. W. Hambley, Dalton Trans., 2007, 3983-3990. 
12 G. L. Lu, R. J. Stevenson, J. Y. C. Chang, P. J. Brothers, D. C. Ware, W. R. Wilson, W. A. Denny and M. Tercel, Bioorg. Med. Chem., 2011, 19, 4861-4867.

13 A. K. Renfrew, N. S. Bryce and T. W. Hambley, Chem. Sci., 2013, 4, 3731-3739.

14 T. W. Failes, C. Cullinane, C. I. Diakos, N. Yamamoto, J. G. Lyons and T. W. Hambley, Chem. - Eur. J., 2007, 13, 2974-2982.

15 G. O. Ahn, D. C. Ware, W. A. Denny and W. R. Wilson, Radiat. Res., 2004, 162, 315-325.

16 J. Y. C. Chang, G. L. Lu, R. J. Stevenson, P. J. Brothers, G. R. Clark, K. J. Botting, D. M. Ferry, M. Tercel, W. R. Wilson, W. A. Denny and D. C. Ware, Inorg. Chem., 2013, 52, 7688-7698.

17 G. O. Ahn, K. J. Botting, A. V. Patterson, D. C. Ware, M. Tercel and W. R. Wilson, Biochem. Pharmacol., 2006, 71, 1683-1694.

18 M. Alimi, A. Allam, M. Selkti, A. Tomas, P. Roussel, E. Galardon and I. Artaud, Inorg. Chem., 2012, 51, 9350-9356.

19 B. J. Kim, T. W. Hambley and N. S. Bryce, Chem. Sci., 2011, 2, 2135-2142.

20 L. Kelland, Nat. Rev. Cancer, 2007, 7, 573-584.

21 K. R. Barnes, A. Kutikov and S. J. Lippard, Chem. Biol., 2004, 11, 557-564.

22 C. Barbara, P. Orlandi, G. Bocci, A. Fioravanti, A. Di Paolo, G. Natale, M. Del Tacca and R. Danesi, Eur. J. Pharmacol., 2006, 549, 27-34.

23 S. Mukhopadhyay, C. M. Barnes, A. Haskel, S. M. Short, K. R. Barnes and S. J. Lippard, Bioconjugate Chem., 2008, 19, 39-49.

24 S. Dhar and S. J. Lippard, Proc. Natl. Acad. Sci. U. S. A., 2009, 106, 22199-22204.

25 C. K. Chen, J. Z. Zhang, J. B. Aitken and T. W. Hambley, J. Med. Chem., 2013, 56, 8757-8764.

26 E. Wexselblatt and D. Gibson, J. Inorg. Biochem., 2012, 117, 220-229.

27 W. H. Ang, I. Khalaila, C. S. Allardyce, L. Juillerat-Jeanneret and P. J. Dyson, J. Am. Chem. Soc., 2005, 127, 1382-1383.

28 M. D. Hall, G. J. Foran, M. Zhang, P. J. Beale and T. W. Hambley, J. Am. Chem. Soc., 2003, 125, 7524-7525.

29 D. M. Griffith, B. Duff, K. Y. Suponitsky, K. Kavanagh, M. P. Morgan, D. Egan and C. J. Marmion, J. Inorg. Biochem., 2011, 105, 793-799.

30 J. P. Parker, H. Nimir, D. M. Griffith, B. Duff, A. J. Chubb, M. P. Brennan, M. P. Morgan, D. A. Egan and C. J. Marmion, J. Inorg. Biochem., 2013, 124, 70-77.

31 E. Meggers, Chem. Commun., 2009, 1001-1010.

32 C. S. Allardyce, P. J. Dyson, D. J. Ellis, P. A. Salter and R. Scopelliti, J. Organomet. Chem., 2003, 668, 35-42.

33 G. S. Smith and B. Therrien, Dalton Trans., 2011, 40, 10793-10800.

34 S. J. Dougan and P. J. Sadler, Chimia, 2007, 61, 704-715.

35 A. Renfrew, Chimia, 2009, 63, 217-219.

36 A. Kurzwernhart, W. Kandioller, C. Bartel, S. Bachler, R. Trondl, G. Muhlgassner, M. A. Jakupec, V. B. Arion, D. Marko, B. K. Keppler and C. G. Hartinger, Chem. Commun., 2012, 48, 4839-4841.
37 W. Kandioller, E. Balsano, S. M. Meier, U. Jungwirth, S. Goschl, A. Roller, M. A. Jakupec, W. Berger, B. K. Keppler and C. G. Hartinger, Chem. Commun., 2013, 49, 3348-3350.

38 C. A. Vock, W. H. Ang, C. Scolaro, A. D. Phillips, L. Lagopoulos, L. Juillerat-Jeanneret, G. Sava, R. Scopelliti and P. J. Dyson, J. Med. Chem., 2007, 50, 2166-2175.

39 K. J. Kilpin, C. M. Clavel, F. Edafe and P. J. Dyson, Organometallics, 2012, 31, 7031-7039.

40 A. A. Nazarov, D. Gardini, M. Baquie, L. Juillerat-Jeanneret, T. P. Serkova, E. P. Shevtsova, R. Scopelliti and P. J. Dyson, Dalton Trans., 2013, 42, 2347-2350.

41 S. Chatterjee, I. Biondi, P. J. Dyson and A. Bhattacharyya, J. Biol. Inorg. Chem., 2011, 16, 715-724.

42 L. K. Filak, S. Goschl, P. Heffeter, K. G. Samper, A. E. Egger, M. A. Jakupec, B. K. Keppler, W. Berger and V. B. Arion, Organometallics, 2013, 32, 903-914.

43 G. Muhlgassner, C. Bartel, W. F. Schmid, M. A. Jakupec, V. B. Arion and B. K. Keppler, J. Inorg. Biochem., 2012, 116, 180-187.

44 A. Castonguay, C. Doucet, M. Juhas and D. Maysinger, J. Med. Chem., 2012, 55, 8799-8806.

45 J. Kljun, A. K. Bytzek, W. Kandioller, C. Bartel, M. A. Jakupec, C. G. Hartinger, B. K. Keppler and I. Turel, Organometallics, 2011, 30, 2506-2512.

46 E. Iniguez, A. Sanchez, M. A. Vasquez, A. Martinez, J. Olivas, A. Sattler, R. A. Sanchez-Delgado and R. A. Maldonado, J. Biol. Inorg. Chem., 2013, 18, 779-790.

47 R. A. Sanchez-Delgado, M. Navarro, K. Lazardi, R. Atencio, M. Capparelli, F. Vargas, J. A. Urbina, A. Bouillez, A. F. Noels and D. Masi, Inorg. Chim. Acta, 1998, 276, 528-540.

48 S. Urig, K. Fritz-Wolf, R. Reau, C. Herold-Mende, K. Toth, E. Davioud-Charvet and K. Becker, Angew. Chem., Int. Ed., 2006, 45, 1881-1886.

49 I. Ott, X. H. Qian, Y. F. Xu, D. H. W. Vlecken, I. J. Marques, D. Kubutat, J. Will, W. S. Sheldrick, P. Jesse, A. Prokop and C. P. Bagowski, J. Med. Chem., 2009, 52, 763-770.

50 S. M. Clardy, D. G. Allis, T. J. Fairchild and R. P. Doyle, Expert Opin. Drug Delivery, 2011, 8, 127-140.

51 S. Mundwiler, B. Spingler, P. Kurz, S. Kunze and R. Alberto, Chem. - Eur. J., 2005, 11, 4089-4095.

52 P. Ruiz-Sanchez, C. Konig, S. Ferrari and R. Alberto, J. Biol. Inorg. Chem., 2011, 16, 33-44.

53 P. Ruiz-Sanchez, S. Mundwiler, B. Spingler, N. R. Buan, J. C. Escalante-Semerena and R. Alberto, J. Biol. Inorg. Chem., 2008, 13, 335-347.

54 M. T. Q. Tran, E. Furger and R. Alberto, Org. Biomol. Chem., 2013, 11, 3247-3254.

55 R. Mukherjee, E. G. Donnay, M. A. Radomski, C. Miller, D. A. Redfern, A. Gericke, D. S. Damron and N. E. Brasch, Chem. Commun., 2008, 3783-3785.

56 U. Schatzschneider, Eur. J. Inorg. Chem., 2010, 1451-1467.

57 L. Zayat, C. Calero, P. Albores, L. Baraldo and R. Etchenique, J. Am. Chem. Soc., 2003, 125, 882-883.

58 L. Zayat, O. Filevich, L. M. Baraldo and R. Etchenique, Philos. Trans. R. Soc., A, 2013, 371. 
59 V. Nikolenko, R. Yuste, L. Zayat, L. M. Baraldo and R. Etchenique, Chem. Commun., 2005, 1752-1754.

60 M. Salierno, E. Marceca, D. S. Peterka, R. Yuste and R. Etchenique, J. Inorg. Biochem., 2010, 104, 418-422.

61 R. N. Garner, J. C. Gallucci, K. R. Dunbar and C. Turro, Inorg. Chem., 2011, 50, 9213-9215.

62 M. A. Sgambellone, A. David, R. N. Garner, K. R. Dunbar and C. Turro, J. Am. Chem. Soc., 2013, 135, 11274-11282.

63 T. Respondek, R. N. Garner, M. K. Herroon, I. Podgorski, C. Turro and J. J. Kodanko, J. Am. Chem. Soc., 2011, 133, 17164-17167.

64 S. Banerjee, P. Prasad, A. Hussain, I. Khan, P. Kondaiah and A. R. Chakravarty, Chem. Commun., 2012, 48, 7702-7704.

65 T. K. Goswami, S. Gadadhar, B. Gole, A. A. Karande and A. R. Chakravarty, Eur. J. Med. Chem., 2013, 63, 800-810.

66 A. Hussain, K. Somyajit, B. Banik, S. Banerjee, G. Nagaraju and A. R. Chakravarty, Dalton Trans., 2013, 42, 182-195.

67 K. Bian and F. Murad, Front. Biosci., 2003, 8, D264-D278. 68 S. P. Fricker, Met. Ions Biol. Syst., 2004, 41, 421-480.

69 B. Heilman and P. K. Mascharak, Philos. Trans. R. Soc., A, 2013, 371.

70 P. T. Burks, J. V. Garcia, R. GonzalezIrias, J. T. Tillman, M. T. Niu, A. A. Mikhailovsky, J. P. Zhang, F. Zhang and P. C. Ford, J. Am. Chem. Soc., 2013, 135, 18145-18152.

71 B. J. Heilman, J. St John, S. R. J. Oliver and P. K. Mascharak, J. Am. Chem. Soc., 2012, 134, 11573-11582.

72 T. R. Johnson, B. E. Mann, J. E. Clark, R. Foresti, C. J. Green and R. Motterlini, Angew. Chem., Int. Ed., 2003, 42, 3722-3729.

73 B. E. Mann, T. R. Johnson, J. E. Clark, R. Foresti, C. Green and R. Motterlini, J. Inorg. Biochem., 2003, 96, 40.

74 J. Niesel, A. Pinto, H. W. P. N'Dongo, K. Merz, I. Ott, R. Gust and U. Schatzschneider, Chem. Commun., 2008, 1798-1800.
75 H. Maeda, Adv. Enzyme Regul., 2001, 41, 189-207.

76 K. K. Coti, M. E. Belowich, M. Liong, M. W. Ambrogio, Y. A. Lau, H. A. Khatib, J. I. Zink, N. M. Khashab and J. F. Stoddart, Nanoscale, 2009, 1, 16-39.

77 J. Della Rocca, D. M. Liu and W. B. Lin, Acc. Chem. Res., 2011, 44, 957-968.

78 J. Panyam and V. Labhasetwar, Adv. Drug Delivery Rev., 2003, 55, 329-347.

79 G. Wenz, Clin. Drug Invest., 2000, 19, 21-25.

80 B. Therrien, G. Suss-Fink, P. Govindaswamy, A. K. Renfrew and P. J. Dyson, Angew. Chem., Int. Ed., 2008, 47, 3773-3776.

81 N. P. E. Barry, O. Zava, P. J. Dyson and B. Therrien, Chem. - Eur. J., 2011, 17, 9669-9677.

82 A. Pitto-Barry, O. Zava, P. J. Dyson, R. Deschenaux and B. Therrien, Inorg. Chem., 2012, 51, 7119-7124.

83 F. Schmitt, J. Freudenreich, N. P. E. Barry, L. JuilleratJeanneret, G. Suss-Fink and B. Therrien, J. Am. Chem. Soc., 2012, 134, 754-757.

84 O. Zava, J. Mattsson, B. Therrien and P. J. Dyson, Chem. - Eur. J., 2010, 16, 1428-1431.

85 L. E. H. Paul, J. Furrer and B. Therrien, J. Organomet. Chem., 2013, 734, 45-52.

86 J. E. M. Lewis, E. L. Gavey, S. A. Cameron and J. D. Crowley, Chem. Sci., 2012, 3, 778-784.

87 N. Metzler-Nolte, Chimia, 2007, 61, 736-741.

88 A. Monney and M. Albrecht, Coord. Chem. Rev., 2013, 257, 2420-2433.

89 L. M. Kaminskas, V. M. McLeod, C. J. H. Porter and B. J. Boyd, Mol. Pharmaceutics, 2012, 9, 355-373.

90 M. E. Davis, Z. Chen and D. M. Shin, Nat. Rev. Drug Discovery, 2008, 7, 771-782. 